\title{
Optofluidic evanescent dye laser based on a distributed feedback circular grating
}

\author{
Wuzhou Song, ${ }^{1}$ Andreas E. Vasdekis, $\left.{ }^{1, a}\right)$ Zhenyu Li, $^{2}$ and Demetri Psaltis ${ }^{1}$ \\ ${ }^{1}$ Optics Laboratory, School of Engineering, Swiss Federal Institute of Technology Lausanne (EPFL), \\ CH-1015 Lausanne, Switzerland \\ ${ }^{2}$ Electrical Engineering, California Institute of Technology, 1200 East California Boulevard, Pasadena, \\ California 91125, USA
}

(Received 20 February 2009; accepted 2 April 2009; published online 24 April 2009)

\begin{abstract}
We demonstrate an optofluidic evanescent laser based on a solid circular distributed feedback grating with the dye solution acting as the cladding layer. The laser mode is confined within the grating and experiences optical gain via the interaction between its evanescent component with the dye solution. Above a pump energy of $9.5 \mu \mathrm{J} /$ pulse, the laser exhibited single mode operation at $571 \mathrm{~nm}$. Stable, narrow-linewidth emission was observed for a wide range of fluid refractive indices, even for those lower than of polydimethylsiloxane. We attribute this property to the evanescent coupling of the laser mode with the fluidic gain. () 2009 American Institute of Physics.

[DOI: $10.1063 / 1.3124652]$
\end{abstract}

Optofluidics have recently attracted substantial attention due to the potential of integrating optics with microfluidics and thus enabling cost-effective methods for lab-on-a-chip applications and noninvasive diagnostics. ${ }^{1,2}$ As such, optofluidic dye lasers are particularly attractive for spectroscopic applications due to the wide choice of emission wavelengths and the possibility of avoiding photobleaching by flowing the gain medium. To this end, various cavity configurations have been demonstrated, such as Fabry-Perot, ${ }^{3}$ microdroplets, ${ }^{4}$ microcavities, ${ }^{5}$ Bragg gratings,${ }^{6,7}$ and photonic crystal fibers. ${ }^{8}$ In all aforementioned structures, the guided mode is confined inside the fluid gain medium and thus the refractive index of the solution determines the emission wavelength. Although this enables the tuning of the lasing wavelength by fluidic mixing, in practice stable and single mode operation require high control of the refractive index of the fluid and moderate flow rates across the waveguides. In addition, due to the relatively high index of polydimethylsiloxane $(\mathrm{PDMS}) \quad\left(n_{\mathrm{PDMS}}=1.4218\right)$, several buffers cannot be directly employed for waveguiding in PDMS based optofluidic lasers.

To overcome this, evanescent field dye lasers have been proposed in the past. ${ }^{9,10}$ In these lasers, the liquid gain medium surrounds a solid waveguide and is optically excited. The excited chromophores in the near-field of the waveguide are evanescently coupled to the laser mode providing the optical gain. To date, several evanescent optofluidic dye lasers have been demonstrated, primarily based on whispering gallery mode resonators such as infilled silica capillaries or fibers embedded in dye solutions. ${ }^{11-13}$ These structures are usually characterized by multimode emission spectra. More recently, evanescent lasers operating in the telecommunication wavelength range have found applications in the field of silicon photonics. ${ }^{14}$

In this letter, we demonstrate an optofluidic evanescent dye laser, exhibiting single mode operation. It comprises of a solid second order circular distributed feedback (DFB) grating and a PDMS chamber filled with dye solution. The thin

${ }^{a)}$ Electronic mail: andreas.vasdekis@epfl.ch. layer of the solution serves as the cladding and covers the entire surface of the solid DFB cavity. Due to the high modal confinement in the solid core, the mode selection and lasing wavelength are primarily determined by the solid DFB cavity. In comparison to liquid core dye lasers, ${ }^{7}$ stable and single mode operation can be achieved for a wide range of refractive indices of the dye solution.

In Fig. 1, a cross-sectional schematic of the circular DFB resonator is illustrated. It is made of the negative photoresist SU-8 (refractive index $n_{\mathrm{SU}-8}=1.59$ ) patterned on top of silicon dioxide layer $\left(n_{\mathrm{SiO}_{2}}=1.46\right)$ on a silicon substrate. The PDMS $\left(n_{\mathrm{PDMS}}=1.4128\right)$ forms a microfluidic chamber which envelops the entire surface of the solid DFB cavity. By infilling the dye solution into the PDMS chamber, a thin layer of liquid gain medium is formed on top of the grating serving as the upper cladding of the cavity. In comparison to linear gratings, the circular DFB cavity has a large surface area and enables an enhanced interaction area of the evanescent tail with the gain medium. The second order DFB grating provides both optical feedback and output coupling via second and first order diffraction, respectively, thus forming a surface emitting optofluidic laser.

The fabrication process is illustrated in Fig. 2. The grating was realized by electron beam lithography (Vistec EBPG5000, $100 \mathrm{kV}$ ). The electron beam resist was a $500 \mathrm{~nm}$ thick film of the negative photoresist SU-8 (GM-1030, Gersteltec Inc., $8 \mu \mathrm{Cb} / \mathrm{cm}^{2}$ ) on a $5 \mu \mathrm{m}$ wet oxide layer on a

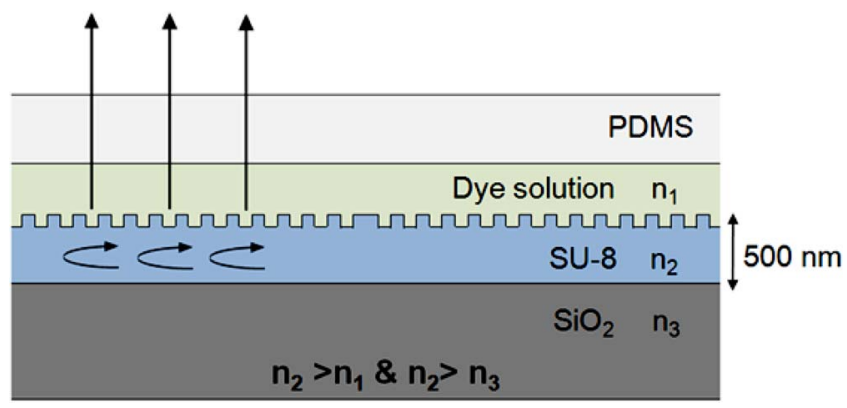

FIG. 1. (Color online) The schematic cross section of the dye laser chip. 


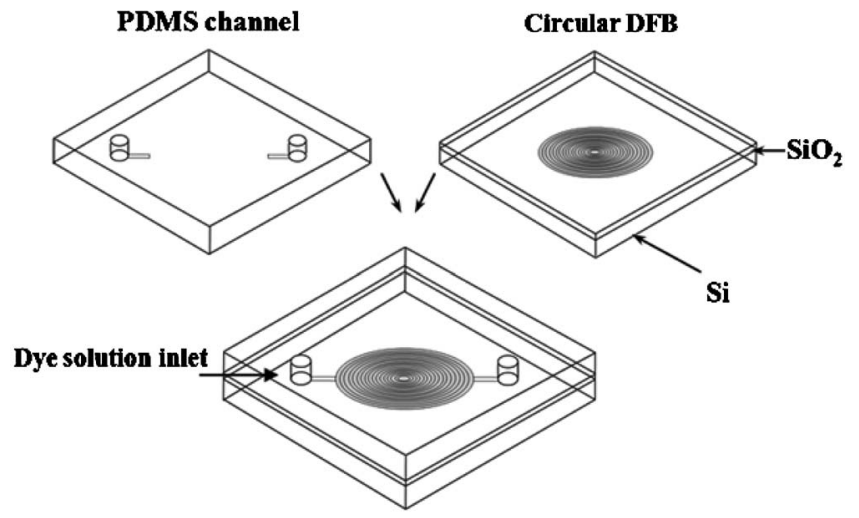

FIG. 2. The fabrication process of the evanescent dye optofluidic laser.

silicon wafer. SU-8 was selected for its optical transparency in the visible spectrum and its high electron beam sensitivity, thus allowing rapid patterning of large area structures. The grating period was $370 \mathrm{~nm}$ in order to satisfy the second order Bragg condition $\Lambda=\lambda / n_{\text {eff }}$, where $n_{\text {eff }}$ is the effective refractive index of the waveguide mode, $\Lambda$ is the grating period and $\lambda$ the emission wavelength. A scanning electron microscopy (SEM) image of the grating at a tilt angle of $30^{\circ}$ is shown in Fig. 3. The width of the grating grooves was approximately $130 \mathrm{~nm}$. The PDMS structure for the microfluidic channel was prepared by replica molding. The master mold for the microfluidic channels was defined using UV lithography on a $4 \mu \mathrm{m}$ thick SU-8 film (GM-1040, Gersteltec Inc.) on a silicon wafer. After oxygen plasma treatment (15 W, $20 \mathrm{~s}$ ), the PDMS layer and the substrate with the circular grating were aligned under a microscope and brought in contact to permanently bond. As shown in Fig. 2, the PDMS layer had no structure in the area corresponding to the grating cavity, except for the two channels that enabled fluidic connection. The dye solution could flow and fill the clearance between the grating and PDMS layer because of the elasticity of PDMS and the absence of bonding between PDMS and SU-8. In this way, the circular grating was covered entirely with a thin fluidic layer. This microfluidic configuration was implemented to minimize the dye solution volume and thus to decrease the background fluorescence which would hinder the detection of stimulated emission. Such configuration proved adequate for the evanescent dye

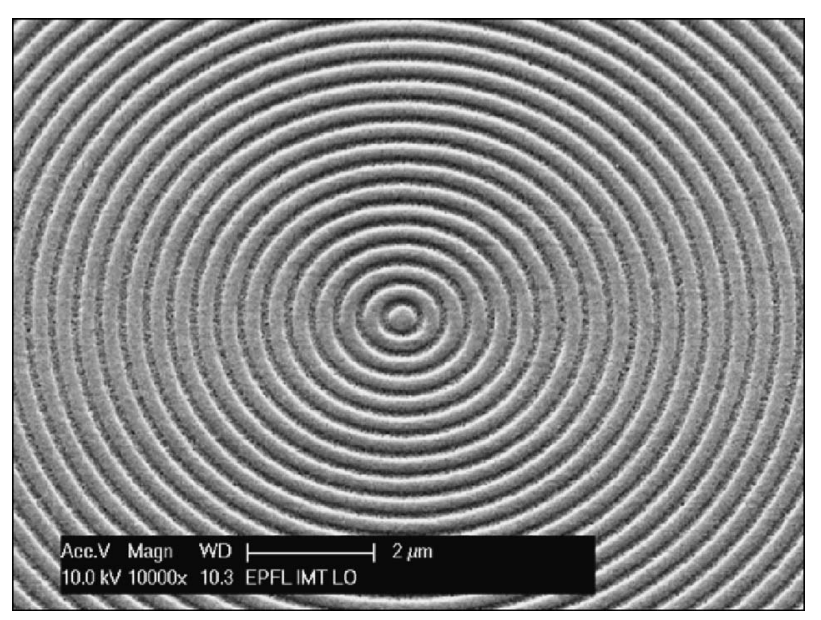

FIG. 3. The SEM image of the circular grating at tilt angle of $30^{\circ}$.

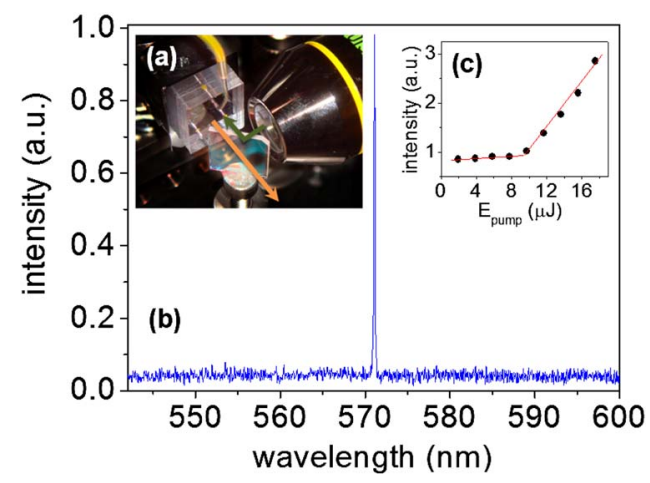

FIG. 4. (Color online) (a) The close-view of experimental set-up. (b) A typical laser emission spectrum above threshold and the input-output relationship (c).

laser operation due to the near-field nature and thus very short penetration depth of the evanescent waves into the fluid. A rhodamine $6 G$ solution was used at a concentration of $1 \mathrm{mg} / \mathrm{ml}$. The solvent was prepared by mixing water and dimethylsulfoxide at a ratio of 54:46 and had a refractive index of 1.401 lower than that of SU-8.

The optofluidic evanescent dye laser chip was optically pumped with a $Q$-switched Nd:YAG laser $(532 \mathrm{~nm}, 4.5 \mathrm{~ns}$ pulses, and $100 \mathrm{~Hz}$ repetition rate). The experimental setup is shown in the inset of Fig. 4(a). The pump laser beam was focused through a $10 \times$ magnification objective lens, reflected by a dichroic mirror (Chroma Inc.) in front of the chip and focused on the laser cavity. The laser emission was normal to the cavity surface. After passing through the same dichroic mirror, it was collected by a fiber coupled charge coupled device spectrometer (Ocean Optics HR4000, $1.2 \AA$ resolution). A typical single mode laser spectrum above threshold is shown in Fig. 4(b). The lasing mode appeared at the wavelength $571.09 \mathrm{~nm}$ and had a linewidth of less than $0.2 \mathrm{~nm}$. A plot of the laser output versus the pump energy is shown in Fig. 4(c). The threshold was estimated to be $9.5 \mu \mathrm{J} /$ pulse. This value is higher than previous reports on optofluidic lasers. ${ }^{6,73,15-17}$ We attribute this primarily to the short absorption length of the pump light due to the top pumping configuration, but also to the reduced overlap of the laser mode with the gain medium.

From the emission wavelength $\lambda$ and the grating period we deduced the waveguide effective index to be $n_{\text {eff }}=1.546$. Based on a one-dimensional (1D) slab waveguide model, the refractive index of SU- 8 was calculated to be $n_{\text {core }}=1.585^{18}$ In addition, the emission spectrum was found to be stable both in intensity and wavelength. There was no detectable spectral shift in the lasing wavelength during the experiment, even when there was rapid dye flow through the cavity. The fluctuation of the output intensity was also below $10 \%$, comparable to the fluctuation of the pump laser.

Due to the high index contrast between the fluid cladding and the solid core, this dye laser maintained single mode operation over a wide range of the dye solution's refractive index. To explore the effect of the fluid refractive index on the emission wavelength, dye solutions of different refractive indices were employed on the same laser chip $(\Lambda=370 \mathrm{~nm})$. We observed a decrease in the emission wavelength for decreasing fluidic indices, as plotted in square dots in Fig. 5(a). Such behavior is expected due to the dependence of the effective mode index on the cladding refractive 

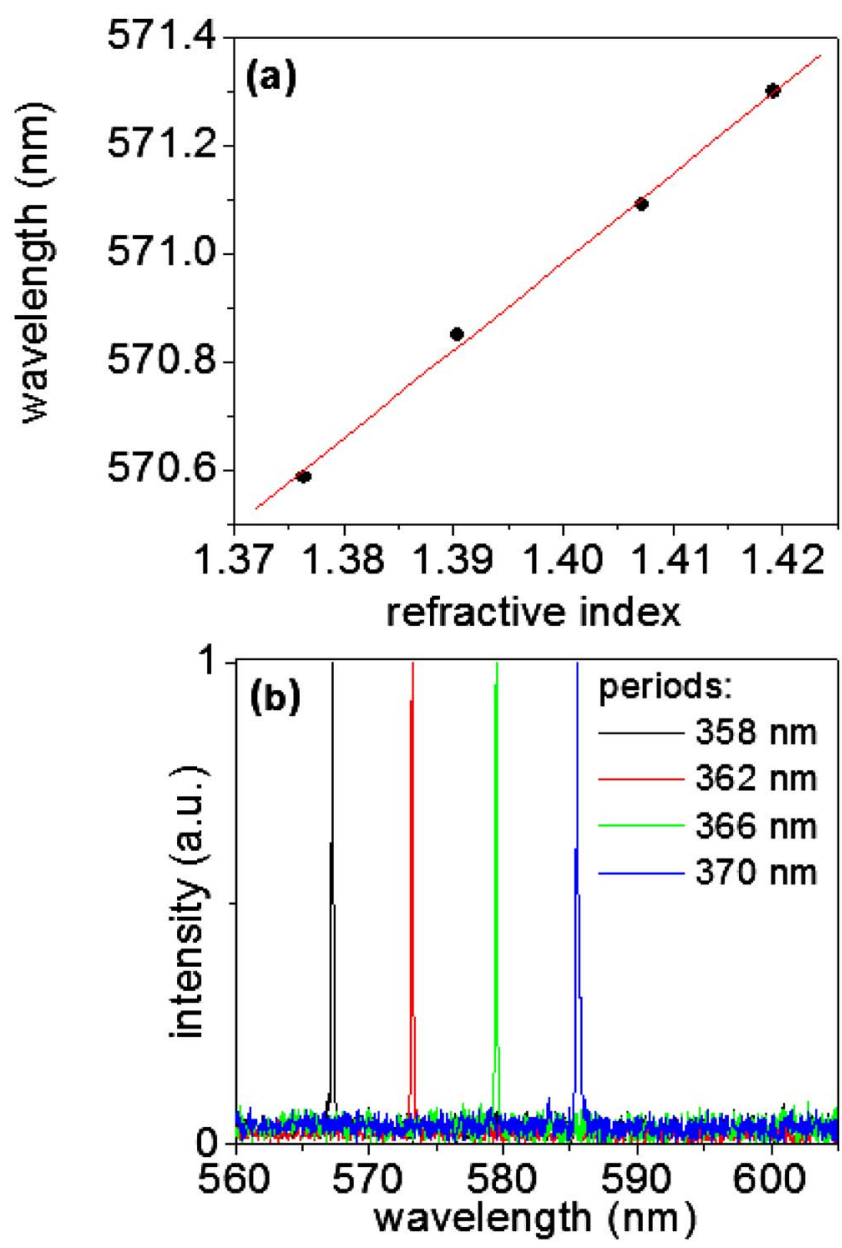

FIG. 5. (Color online) (a) The lasing wavelength vs the refractive index of the dye solution. The square dots represent the experimental data and the line indicates the simulation result. (b) The emission spectra of evanescent optofluidic dye laser of different grating periods.

index. The experimental results were found to agree with calculations based on a 1D slab waveguide model [straight line in Fig. 5(a)], further confirming that the laser mode is confined within the solid waveguide. The variation in the cladding index also induces a change in the confinement factor of the laser mode. However, this was not manifested in the laser threshold in our experiments due to the simultaneous tuning of the emission wavelength and thus the different gain that rhodamine $6 G$ exhibits at these wavelengths. In addition, we did not observe laser emission from dye solutions with refractive indices below $n=1.35$, although waveguide modes are supported for these indices. This is attributed to the high concentration of water in the solution, which has been shown to degrade the gain properties of rhodamine $6 G .{ }^{19}$ Finally, we investigated the tuning of the emission wavelength by changing the period of the circular DFB grating. The emission spectra are plotted in Fig. 5(b) for the grating periods of $358,362,366$, and $370 \mathrm{~nm}$, denoting a tuning range of approximately $20 \mathrm{~nm}$.

In summary, we demonstrated an optofluidic evanescent dye laser based on a solid circular DFB grating. Under optical excitation, gain was provided by the interaction of the evanescent component of the waveguide mode with the liquid cladding. The integration of a strongly wavelength selective periodic structure enabled single mode operation. Due to the evanescent coupling between the laser mode and the fluid, single mode operation was maintained for a wide range of refractive indices of the fluid. This evanescent laser was realized by constructing an optofluidic circuit on a pretreated silicon wafer. Further integration of electrodes, or absorbing layers can be implemented on top of the cladding layer for passive or active modulation, since in such evanescent wave coupled gain lasers these integration steps would induce minimal modal perturbation. ${ }^{11,14,20}$ In future work, we will investigate ways to reduce the lasing threshold by modifying the pumping geometry and employing first order gratings, which we have shown in the past to reduce the lasing threshold. ${ }^{16}$

The research was partially supported by the DARPA center for optofluidic integration.

${ }^{1}$ D. Psaltis, S. R. Quake, and C. H. Yang, Nature (London) 442, 381 (2006).

${ }^{2}$ C. Monat, P. Domachuk, and B. J. Eggleton, Nat. Photonics 1, 106 (2007).

${ }^{3}$ B. Helbo, A. Kristensen, and A. Menon, J. Micromech. Microeng. 13, 307 (2003).

${ }^{4}$ S. X. Qian, J. B. Snow, H. M. Tzeng, and R. K. Chang, Science 231, 486 (1986).

${ }^{5}$ H. Yokoyama, Science 256, 66 (1992).

${ }^{6}$ M. Gersborg-Hansen and A. Kristensen, Appl. Phys. Lett. 89, 103518 (2006).

${ }^{7}$ Z. Y. Li, Z. Y. Zhang, T. Emery, A. Scherer, and D. Psaltis, Opt. Express 14, 696 (2006).

${ }^{8}$ A. E. Vasdekis, G. E. Town, G. A. Turnbull, and I. D. W. Samuel, Opt. Express 15, 3962 (2007).

${ }^{9}$ E. P. Ippen and C. V. Shank, Appl. Phys. Lett. 21, 301 (1972).

${ }^{10}$ G. Pendock, H. S. Mackenzie, and F. P. Payne, Electron. Lett. 28, 149 (1992).

${ }^{11}$ H. J. Moon, Y. T. Chough, and K. An, Phys. Rev. Lett. 85, 3161 (2000).

${ }^{12}$ X. S. Jiang, Q. H. Song, L. Xu, J. Fu, and L. M. Tong, Appl. Phys. Lett. 90, 233501 (2007).

${ }^{13}$ S. I. Shopova, H. Y. Zhou, X. D. Fan, and P. Zhang, Appl. Phys. Lett. 90 221101 (2007).

${ }^{14}$ B. R. Koch, A. W. Fang, O. Cohen, and J. E. Bowers, Opt. Express 15, 11225 (2007)

${ }^{15}$ S. Lacey, I. M. White, Y. Sun, S. I. Shopova, J. M. Cupps, P. Zhang, and X. D. Fan, Opt. Express 15, 15523 (2007).

${ }^{16}$ W. Z. Song, A. E. Vasdekis, Z. Y. Li, and D. Psaltis, Appl. Phys. Lett. 94, 051117 (2009).

${ }^{17}$ C. Peroz, J. C. Galas, L. Le Gratiet, Y. Chen, and J. Shi, Appl. Phys. Lett. 89, 243109 (2006).

${ }^{18}$ R. G. Hunsperger, Integrated Optics (Springer, Berlin, 2002).

${ }^{19}$ K. Igarashi, M. Maeda, T. Takao, M. Uchiumi, Y. Oki, and K. Shimamoto, Jpn. J. Appl. Phys., Part 1 34, 3093 (1995).

${ }^{20}$ E. B. Namdas, M. Tong, P. Ledochowitsch, S. R. Mednick, J. D. Yuen, D. Moses, and A. J. Heeger, Adv. Mater. (Weinheim, Ger.) 21, 799 (2009). 